\title{
The Ekman Spiral for a Linearly Varying Eddy Viscosity
}

\author{
F. C. W. OLSON**
}

For the past fifty years, the Ekman Spiral has occupied a prominent place in theoretical oceanography. Since the Spiral seems to be elusive when sought in the ocean, it is easy to conclude that the mathematical elegance in the theoretical treatment is responsible in part for the continued popularity which the Ekman Spiral enjoys. The purpose of this note is to call attention to another mathematically elegant treatment of the spiral for the case where the eddy viscosity is assumed to vary linearly with the depth and the depth is finite. Of particular interest is the close analogy between this problem and that of "skin effect" in a cylindrical conductor carrying an alternating current.

Following the classical derivation of the Ekman Spiral, we shall take the equations of motion in the form given by Sverdrup et al (1942):

$$
\begin{aligned}
& \frac{\rho v}{c}+\frac{d}{d z}\left(A \frac{d u}{d z}\right)=0 \\
& -\frac{\rho u}{c}+\frac{d}{d z}\left(A \frac{d v}{d z}\right)=0
\end{aligned}
$$

and we shall assume that the eddy viscosity is given by

$$
A=a(d-z)
$$

where

$$
\begin{aligned}
& a=\text { constant } \\
& c=\frac{1}{2 \omega \sin \varphi} \\
& \phi=\text { latitude (assumed constant) } \\
& \omega=\text { angular velocity of the earth } \\
& d=\text { depth of water } \\
& \rho=\text { density }
\end{aligned}
$$

$x, y, z$ left handed coordinate system with $z$ measured from the sur-

* Received Jan. 26, 1959.

* U. S. Navy Mine Defense Laboratory Panama City, Florida face downward

$u, v$ velocity components in the $x, y$ direction

We shall further assume that the wind stress $\tau_{a}$ is directed along the $y$ axis and that

$$
\begin{aligned}
& \left(A \frac{d v}{d z}\right)_{z=0}=-\tau a \\
& \left(A \frac{d u}{d z}\right)_{z=0}=0
\end{aligned}
$$

On making the substitution

$$
z=d-\frac{a c \zeta^{2}}{4 \rho}
$$

the following equations are obtained from equations (1), (2), (3), (4) and (5):

$$
\begin{gathered}
v+\frac{1}{\zeta} \frac{d u}{d \zeta}+\frac{d^{2} u}{d \zeta^{2}}=0 \\
-u+\frac{1}{\zeta} \frac{d v}{d \zeta}+\frac{d^{2} v}{d \zeta^{2}}=0 \\
\left(\frac{d v}{d \zeta}\right)_{\zeta o}=\frac{\tau a c \zeta_{0}}{2 \rho d} \\
\left(\frac{d u}{d \zeta}\right)_{\zeta o}^{\prime}=0
\end{gathered}
$$

where

$$
\zeta_{0}=(4 \rho d / a c)^{\frac{1}{2}}
$$

corresponds to $z=0$.

Multiplying (8) by $i=\sqrt{-1}$, adding this to (7) and setting

$$
V=u+i v
$$

we obtain

$$
\frac{d^{2} V}{d \zeta^{2}}+\frac{1}{\zeta} \frac{d V}{d \zeta}-i V=0
$$

whose solution is (McLachlan, 1955, page 190*)

$$
V=C_{1} J_{0}\left(\zeta i^{\frac{3}{2}}\right)+C_{2} K_{0}\left(\zeta i^{\frac{1}{2}}\right)
$$

Since the velocity at the bottom $(\zeta=0)$ must be finite, $C_{2}=0$. The solution may then be written

* The notation and treatment of Bessel functions in this paper follows closely that given by McLachlan. For this'reason no explanatory material will be given here. 


$$
\begin{aligned}
V=C_{1} J_{0}\left(\zeta i^{\frac{3}{2}}\right) & =C_{1}(\text { ber } \zeta+i \text { bei } \zeta) \\
& =C_{1} M_{0}(\zeta) e^{i \theta_{0}(\zeta)}
\end{aligned}
$$

The boundary conditions (9) and (10) may be expressed as

$$
\left(\frac{d V}{d \zeta}\right)_{\zeta o}=\left(\frac{d u}{d \zeta}+i \frac{d v}{d \zeta}\right)_{\zeta_{0}}=i \frac{\tau a c \zeta_{0}}{2 \rho d}
$$

we have then

$$
\begin{aligned}
\left(\frac{d V}{d \zeta}\right)_{\zeta_{0}} & =C_{1}\left(\operatorname{ber}^{\prime} \zeta+i \text { bei }^{\prime} \zeta\right)_{\zeta_{0}} \\
& =C_{1} M_{1}\left(\zeta_{0}\right) e^{i\left[\theta_{1}\left(\zeta_{0}\right)-\frac{\pi}{4}\right]}=i \frac{\tau a c \zeta_{0}}{2 \rho d}
\end{aligned}
$$

Solving for $C_{1}$ and substituting in (15) we get

where

$$
V=V_{0} \frac{\zeta_{0}}{2} \frac{M_{0}(\zeta)}{M_{1}\left(\zeta_{0}\right)} e^{i\left[\theta_{0}(\zeta)-\theta_{1}\left(\zeta_{0}\right)+\frac{3 \pi}{4}\right]}
$$

$$
V_{0}=\frac{\tau_{a} c}{\rho d}
$$

Since: $\zeta_{0} M_{0}\left(\zeta_{0}\right) / 2 M_{1}\left(\zeta_{0}\right)$ approaches unity as $\zeta_{0}$ approaches zero (coorresponding to $z=d$ ), $V_{0}$ may be interpreted as the velocity at the bottom. we could also write (18) as

where

$$
V=V_{1} \frac{M_{0}(\zeta)}{M_{0}\left(\zeta_{0}\right)} e^{i\left[\theta_{0}(\zeta)-\theta_{1}\left(\zeta_{0}\right)+\frac{3 \pi}{4}\right]}
$$

$$
V_{1}=\frac{\tau a c \zeta_{0} M_{0}\left(\zeta_{0}\right)}{2 \rho d M_{1}\left(\zeta_{0}\right)}=\tau_{a} \sqrt{\frac{c}{\rho A_{0}}} \frac{M_{0}\left(\zeta_{0}\right)}{M_{1}\left(\zeta_{0}\right)}
$$

and $\mathrm{A}_{0}=$ ad.

In this case, $V_{1}$ is the surface velocity. Inspection of $(18 \mathrm{c})$ reveals that the surface velocity increases as the depth decreases.

The transport $T$ is obtained by integrating (18) with respect to $z$ from $z=0$ to $z=d$. Doing so we find that

$$
T=\frac{\tau_{a} c}{\rho}
$$

Thus the transport is $90^{\circ}$ cum sole to the wind and its magnitude is just that predicted for the simple Ekman theory (SET).

The angle $\psi$ between the current and the wind is found from (18).

We have

$$
\begin{aligned}
\tan \psi=\frac{u}{v} & =\frac{\cos \left(\theta_{0}-\theta_{1}+\frac{3 \pi}{4}\right)}{\sin \left(\theta_{0}-\theta_{1}+\frac{3 \pi}{4}\right)} \\
& =\tan \left(\theta_{1}-\theta_{0}-\frac{\pi}{4}\right)
\end{aligned}
$$

and

$$
\psi=\theta_{1}\left(\zeta_{0}\right)-\theta_{0}(\zeta)-\frac{\pi}{4}
$$

Since $\zeta$ varies from 0 to $\zeta_{0}$, the total angle through which the current turns from top to bottom is given by $\theta_{0}\left(\zeta_{0}\right)-\theta_{0}(0)=\theta_{0}\left(\zeta_{0}\right)$. Selected values of $\theta_{0}\left(\zeta_{0}\right)$ are given in Column II of Table 1. The angle between the surface current and the wind is

$$
\psi_{0}=\theta_{1}\left(\zeta_{0}\right)-\theta_{0}\left(\zeta_{0}\right)-\frac{\pi}{4}
$$

values of which are given in Column IV of Table 1. For very shallow waters, the surface

Table 1. Selected values of the polar forms of the ber and bei functions.

\begin{tabular}{lcccccc}
\hline I & II & III & IV & V & VI & VII \\
\hline$\zeta$ & $\theta^{\circ}{ }_{0}$ & $\theta^{\circ}{ }_{1}$ & $\theta_{\mathbf{1}}-\theta_{0}-45$ & $M_{0}$ & $M_{\mathbf{1}}$ & $\zeta M_{0} / 2 M_{\mathbf{1}}$ \\
\hline 0 & \multicolumn{1}{c}{0} & 135 & 90 & 1.000 & 0.000 & 1.000 \\
0.1 & 0.14 & 135.07 & 89.93 & 1.000 & 0.0500 & 1.000 \\
0.5 & 3.58 & 136.79 & 88.21 & 1.001 & 0.2500 & 1.001 \\
1 & 14.23 & 142.16 & 82.93 & 1.016 & 0.5013 & 1.014 \\
2 & 52.29 & 163.27 & 65.98 & 1.229 & 1.041 & 1.181 \\
5 & 178.93 & 273.55 & 49.62 & 6.231 & 5.809 & 2.682 \\
10 & 382.10 & 474.28 & 47.18 & 149.8 & 144.7 & 5.18 \\
20 & 787.52 & 878.57 & 46.05 & $1.242 \times 10^{5}$ & $1.220 \times 10^{5}$ & 10.18 \\
45 & 1800.53 & 1890.98 & 45.45 & $3.929 \times 10^{12}$ & $3.899 \times 10^{12}$ & 22.68
\end{tabular}

current approaches a direction $90^{\circ}$ cum sole to the wind. This is perhaps the most striking deviation of this theory from the SET but it is consistent with Fjeldstad (1929) who obtained deviations in excess of $45^{\circ}$ for shallow waters when the eddy viscosity varies as the $3 / 4$ power of the distance from the bottom. For deep waters, $\psi_{0}$ approaches $45^{\circ}$, in agree- 
ment with the SET.

\section{Analogy with the "Skin Effect."}

The phenomenon of the skin effect in the case of a wire carrying an alternating current is well known. It is remarkable that equation (18) describes this phenomenon exactly. Let the radius of the wire be $a, r$ be the distance from the center, $\sigma_{m}$ be the mean current density and $\sigma$ be the current density. McLachlan (1955, page 157) gives the following equation for $\sigma$,

$$
\sigma=\sigma_{m} \frac{k a}{2} \frac{M_{0}(k r)}{M_{1}(k a)} e^{i\left[\theta_{0}(k r)-\theta_{1}(k a)+\frac{3 \pi}{4}\right]}
$$

Comparing (18a) and (19), we see that $V_{0}$ is the mean transport per unit depth, corresponding to mean current density; $\zeta$ corresponds to $k r$ and $\zeta_{0}$ to $k a$. Even the phase relation indicated in the exponent is exactly analogous. Further, $k a$ is the same function of resistivity and frequency as $\zeta_{0}$ is of eddy viscosity and trequency of the earth's rotation.

\section{Acknowledgements.}

The author wishes to thank Dr. K. Yoshida for having pointed out to him not only a factor of 2 which erroneously occurred in the derivation but also a questionable interpretation of equation (18c). Both corrections have been made in this paper. Thanks are also due to Dr. T. Ichiye for his suggestions during the preparation of this paper.

\section{References}

Fjeldstad, J. E., 1929. Ein Beitrag zur Theorie der Winderzeugten Meeresströmungen. Gerlands Beit. z. Geophysik, Bd. 23.

Mclachlan, N. W., 1955. "Bessel Functions for Engineers." Oxford, Clarendon Press.

Sverdrup, H. U., M. W. Johnson and R. H. Fleming, 1942. “The Oceans." New York, Prentice-Hall. 